\title{
La base social de la aldea global. Aproximaciones para entender la naturaleza del mundo virtual
}

\author{
Ernesto Faerron-Chavarría * \\ (iD https://orcid.org/0000-0002-9506-2313 \\ Gustavo Cabezas-Barrientos ** \\ iD https://orcid.org/0000-0003-2859-1554
}

Recibido: 19 de febrero, 2019 • Aceptado: 18 de noviembre, 2019

RESUMEN

Este ensayo propone, a partir de la teoría crítica, una visión de la nueva ciudadanía y de la sociedad que se ha configurado con los medios digitales, así como establecer ciertos comportamientos a la luz de algunos de los puntos teóricos y con la visión occidental de este fenómeno; aspectos

\section{Formato de citación según APA}

Faerron Chavarría, E. y Cabezas Barrientos, G. (2020). La base social de la aldea global. Aproximaciones para entender la naturaleza del mundo virtual. Revista Espiga, 19 (39), 1-10.

Formato de citación según Chicago-Deusto

Faerron Chavarría, Ernesto y Cabezas Barrientos, Gustavo (2020). «La base social de la aldea global. Aproximaciones para entender la naturaleza del mundo virtual». Revista Espiga 19, n. ${ }^{\circ} 39$ (enero-junio, 2020): 1-10.

* Máster en Educación a Distancia E-Learning de la Caribbean International University. Estudiante de la Maestría en Dirección Estratégica de Tecnologías de la Información de la Universidad Internacional Iberoamericana, de Puerto Rico. Licenciado y bachiller en Bibliotecología de la Universidad Nacional de Costa Rica. Coordinador, profesor e investigador de la Cátedra de Tratamiento de la Información de las carreras de Bibliotecología de la Escuela de Ciencias Sociales y Humanidades, de la Universidad Estatal a Distancia de Costa Rica. Intereses de investigación: bibliotecología, bibliometría, cienciometría, sociedad del conocimiento, inteligencia artificial, tecnología y sus implicaciones en la sociedad, web semántica, software libre. Correo: efaerron@uned.ac.cr

** Máster en Criminología, Universidad Estatal a Distancia (UNED), Costa Rica. Licenciado y bachiller en Sociología y Antropología de la Universidad de Costa Rica. Desde 2013, coordinador, profesor e investigador de la Cátedra de Sociología de la Escuela de Ciencias Sociales y Humanidades de la UNED. Intereses de investigación: desigualdad, migraciones, género, política electoral, crecimiento y desarrollo, población urbano-marginal, adicciones, sociedad del conocimiento, inteligencia artificial, tecnología y sus implicaciones en la sociedad. Correo: gcabezas@uned.ac.cr 
mostrados en la ciudadanía dentro de cuatro grandes grupos en las redes sociales que conforman esta aldea global. Además, sugiere una forma de definir la estructura social de las sociedades digitales desde la epistemología crítica.

Palabras clave: Sociedad de la información, ermitaños digitales, screenagers, hikikimoris.

\section{Introducción}

Las sociedades humanas (desde el neolítico y, en especial, por su vulnerabilidad) existen prácticamente a partir de que decidieron reunirse en grupos para sobrevivir; este elemento importa porque al ser un grupo en crecimiento, por consiguiente requiere pensar en una sociedad cada vez más compleja.

Por tanto, hoy más que nunca se debería plantear la interrogante de si, al lado de la sociedad física posmodernista, se está desarrollando una sociedad diferente a través de las Tecnologías de la información y la comunicación. Esta sociedad surge quizá como una comunidad sin territorio, global y, por supuesto, una comunidad ubicua.

Si una sociedad se compone por grupos sociales de una cultura semejante, en un espacio común, entonces se debe entender que las comunidades virtuales ${ }^{1}$ son, de hecho, sociedades que tienen su existencia en un espacio determinado, donde los diferentes grupos interactúan con el fin de construir una colectividad estructurada y estratificada en campos específicos.

Esto no es nada nuevo. Pierre Bordieu había anticipado, en la teoría de campo, que los sujetos sociales manejan sus intereses, su conducta y hasta invierten sus capitales económico, social y cultural en este espacio particular.

A pesar de la resistencia que siempre ha existido de pensar que el espacio virtual no es «real», lo cierto es que el llamado proceso de globalización ha sido posible gracias a la irrupción de las nuevas tecnologías e internet, los cuales han influenciado elementos tales como la forma de vestir y la alimentación ¿Quién no ha intentado hacer un dhai, una tortilla española o cualquier comida que vaya más allá de la comida criolla gracia a los miles de videos de YouTube o de las recetas en línea? Igualmente hay manuales de maquillaje, de trucos para vestir o convertir una pieza de vestimenta ordinaria en algo extraordinario.

Y en todo caso, los resultados son comparados entre grupos que manejan los mismos intereses y afinidades con las nuevas tecnologías, lo que genera una interacción que refuerza los valores compartidos por ese grupo social determinado.

1. Desde el punto de vista de Castells (2001), «...son diferentes de las comunidades del mundo físico, pero no necesariamente menos intensas, o menos efectivas en unir y movilizar». 
Para fines de este ensayo, se hablará del espacio físico como «sociedad análoga», entendida como una sociedad histórica o tradicional propia de una formación socioeconómica; mientras que el espacio virtual como «sociedad digital» o «sociedad virtual», como sociedades existentes en el ciberspacio.

Bordieu da un concepto de campo, en el cual indica que es un espacio donde se llevan a cabo una serie de interacciones dialécticas entre individuos que luchan por privilegiar sus intereses y denigrar los de los otros individuos con intereses diferentes. Estos individuos o agentes crean e interactúan en un campo dialéctico, donde se encuentra en juego un capital simbólico; habrá, entonces, una serie de alianzas, discrepancias, luchas por ese capital simbólico, que es el que les otorga legitimidad, autoridad o prestigio a los agentes que posean este capital. Cada campo tiene sus instituciones, lenguaje y reglas específicas para lograr este capital.

Por ejemplo, el campo de las personas que consumen información en recetas de cocina, los agentes o participantes de ese campo particular tratan, de alguna manera, de obtener reconocimiento, este agente debe pasar por un proceso de aprendizaje que permite tener ciertas estrategias y alianzas que faciliten ese proceso en esa relación dialéctica entre agentes. De esta manera, comparte sus recetas con el propósito de obtener likes ${ }^{2}$ que aumenten su reconocimiento.

Quien logra ese capital simbólico recibe reconocimiento público. Por ejemplo, una persona en una sociedad busca el reconocimiento mediante los siguientes capitales simbólicos ${ }^{3}$ :

a. El reconocimiento recibido por los recursos económicos que tenga (capital económico), ya sea en dinero, bienes, propiedades; de igual manera, criptomonedas, objetos $\mathrm{y}$ experiencia en juegos, dominios de internet, etc.

b. Reconocimiento por el capital social, que es el de individuos de cierto renombre, ya sea por su fama y su capacidad de influir. En los entornos virtuales existen personas que actúan de esa forma como influencers, celebritys, líderes de opinión, bloggers o youtubers ${ }^{4}$.

c. Reconocimiento por su capital cultural, que es el reconocimiento de los logros alcanzados por años de aprendizaje, como los certificados de educación, pregrado, grado y posgrado; de igual manera se tienen medios de reconocimiento como likes, sistemas de puntajes, visualizaciones, etc.

No todas las personas están dispuestas a vivir en estos entornos digitales o virtuales, lo cual no significa que necesariamente viven aislados, pero es claro que hoy la

2. Se refiere a la interacción que implica que una persona que tiene agrado de una publicación en redes sociales.

3. En muchos de estos espacios (digitales o virtuales) se adquieren desde simples trajes o «skins», como en Fortnite, y propiedades en mundos como Second Life; también vale mencionar los patios de juego de Fortnite.

4. Individuos que tienen como modo de vida ser generadores de opinión. 
decisión de esa sociedad análoga (que no está arraigada digitalmente) para interactuar en la sociedad digital está a punto de desaparecer y son muchas personas las que viven literalmente en internet: tienen su empleo, compran su comida, su ropa y hasta tienen sexo virtual. Pero igualmente, si bien reciben realimentación y afirmación en este mundo digital, reciben realimentación negativa que puede terminar con la sanción social que genere una respuesta física como depresión o ansiedad.

Lejos de centrarse en la psique de los que entran y salen de las sociedades digitales, de estos mundos virtuales, se debe entender algunas características de estas sociedades que requieren la atención en este artículo.

En este sentido, si se habla de la sociedad occidental, se entiende el proceso de cambios sociales que han traido hasta este instante de la historia; como sociedad, en un momento histórico ha considerado que el eje fundamental de su historia era una sociedad basada en la creencia de Dios y, esa construcción social, terminó por agotarse hasta desarrollar un renacimiento de la curiosidad que, como seres humanos, ha creado una realidad propia, al ser la humanidad dueña de su destino.

Es en esta era de la modernidad, donde la sociedad análoga ha caminado por tantos siglos, que se colocan, de manera paralela, los ladrillos que cimientan esta sociedad digital. Ada Lovelace fue la primera en contruir un algoritmo para ser procesado por una máquina. Actualmente, la palabra algoritmo ni siquiera suena a ciencia ficción.

\section{La base material, sustento de una sociedad}

Al pensar en una pirámide, la sociedad analógica ha sentado la base sobre la que se han construido los pilares digitales sobre los cuales se sustentan las sociedades digitales del mundo virtual. Una sociedad analógica sienta su base en un modo de producción que impone su base material, en este caso la sociedad capitalista. Sobre esto, contruye valores, normas y creencias que sustenten esa base social, cultura e ideología que dan sentido a la realidad observada. La sociedad digital tiene una estructura muy distinta porque la base de ella no está basada en un modo de producción, sino que se asienta en las tecnologías.

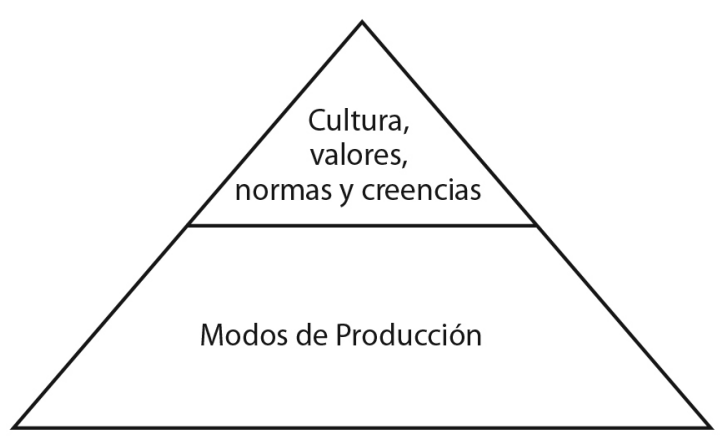

Estructura social de una sociedad analógica

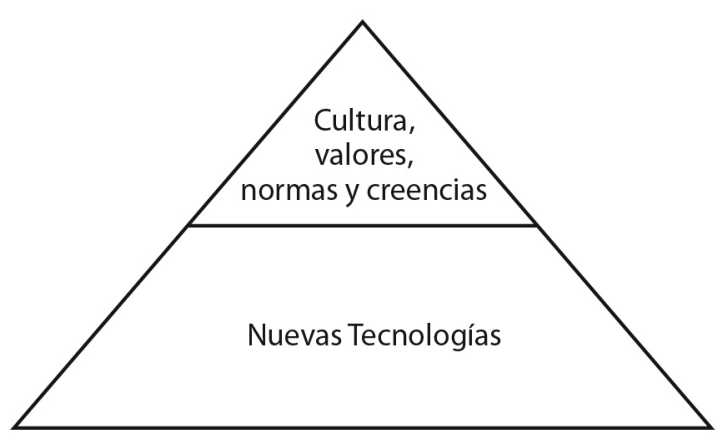

Estructura social de una sociedad digital

Figura 1. Fuente: Elaboración propia. 
Esto es de suma importancia, porque la sociedad grecorromana basaba sus valores y creencias en un modo esclavista de producción, mientras que en la Edad Media eran el feudo y los siervos la base material que daba una existencia de todas las construcciones sociales, como el ideal caballeresco y la fidelidad al monarca. La sociedad capitalista es la que posibilita y da los primeros pasos para crear las tecnologías que permitan la individualidad y la libertad, valores clave en las sociedades digitales.

Se tiene, entonces, que una sociedad se define por su base material y esta sustenta sus valores, normas y creencias. Pero, cuando se habla de sociedades digitales su base material se sustenta en la tecnología y son los avances que tengan estas nuevas tecnologías, los que permiten hacer, como seres humanos; lo que posibilita no solo en la percepción de la realidad y la captura del conocimiento, sino también en el desarrollo de nuevas habilidades sociales que cambian por completo la percepción de la existencia.

Esta base material tecnológica es igualmente un elemento que divide esta sociedad virtual en clases; por ejemplo, el ancho de banda de internet al que se tiene acceso, la tecnología de video, el tipo de procesador, la cantidad de memoria, el acceso al software necesario para la interaccion entre individuos dentro de esta sociedad. Esta idea es la misma que fracciona la sociedad en clases, estratificando y discriminando los grupos sociales de acuerdo con su acceso a bienes en la Revolución Industrial. En esta revolución tecnológica, la dicrimación es mediante el acceso a aquellos servicios o software (o aplicaciones) que permiten interactuar con otros sujetos sociales en la red.

Esta sociedad digital tiene la posibilidad de desdoblarse, cosa que no logra la sociedad análoga, es decir, puede tener una realidad subjetiva y, al mismo tiempo, una realidad objetiva. Es objetiva porque los individuos que la manejan y los límites de esta sociedad los da la tecnología, que existe en esa realidad objetiva. Pero es, al mismo tiempo, una realidad subjetiva, ya que es atemporal, asincrónica, no tiene espacio, se nutre de esa realidad objetiva, pero es libre e individual en el plano subjetivo. Los individuos que trabajan freelance o en teletrabajo, saben muy bien cómo aprovechar estos espacios subjetivos.

Esta realidad subjetiva claramente es una construcción humana, no se niega que se llega a este momento gracias a una transformación cultural, pero se toman en cuenta los ejemplos de Second Life o los Sims, al entender que hay realidades en su interacción humana subjetiva que escapan de la arquitectura inicial y se tornan independientes de ella; es decir, un elemento que los ingenieros ignoraban que ocurriría, a suerte de Deus ex machina.

Ahí es donde está la otra gran diferencia entre ambas sociedades, el pragmatismo tecnológico logra instaurar nuevas formas de convivencia social; así como la sociedad capitalista se impone al feudalismo de manera pragmática y lenta, de la misma forma este pragmatismo tecnológico se impone lentamente sobre los modelos democráticos al cambiar las dinámicas de la realidad objetiva desde la subjetividad tecnológica. Ejemplo de ello es cómo esta realidad subjetiva, llamada genéricamente «redes», se torna de gran relevancia en las elecciones políticas.

Y es que estos modelos democráticos en la realidad objetiva empiezan a dar señales de desgaste, por lo que la subjetividad en la realidad social empieza a imponerse; pero 
no desde la base material de la sociedad, sino desde la base tecnológica, que pone los límites de esta sociedad digital.

En resumen, a criterio de los autores las sociedades digitales se distinguen en que la base material de dicha sociedad está sustentada en las nuevas tecnologías y los límites los imponen estas tecnologías; lo segundo es que frente al pragmatismo de la realidad de la sociedad análoga, se impone en la sociedad digital una visión subjetiva de la realidad. La atemporalidad y la realidad subjetiva son los principales elementos sobre los que se asientan los valores, normas y creencias de estas sociedades, donde la cultura y la ideología se intersecan, se trasforman e hibridan; no son sólidos, diría Zygmunt Bauman, son líquidos. Esto ocurre porque el cambio es el motor de la historia, y estos espacios de libertad, individualidad, de expresión indomable, están generando claros indicios de cambio social, ya sea en esa base material (cadena de bloques), como en la estructura de las interaciones sociales; es decir, en sus valores, normas y creencias. No faltan críticos de ello, que generan resistencia a los cambios.

En un extremo de la recta se tienen individuos que, como el filósofo Enric Puig Punyet, comparan al internet como una adicción y alienta a las personas a vivir más cercano al mundo análogo que al digital. Este filósofo es el abanderado de que las nuevas tecnologías acabarán dominando a las personas mediante el manejo del big data ${ }^{5}$ que se genera cuando se interactúa en la red.

En este sentido, Bauman mencionaba que duda acerca de las ventajas que supondría la era digital, calificaba de trampa las redes sociales, ya que son una prótesis de las capacidades sociales humanas, al distorsionar la forma en que se interactúa y que obliga al individuo a crear su propia comunidad.

El sociólogo polaco afirma que este sustituto de la comunidad surge por la incapacidad de algunos individuos de pertenecer a aquella comunidad que supuestamente ha creado. Se debe entender que en este mundo digital es fácil crear como evitar la controversia; solo falta añadir o borrar amigos con un clic, por lo que no se necesitan grandes habilidades sociales.

Por ello, a muchas personas que buscan no encontrarse en un estado de desamparo total, internet las dota de esta herramienta (prótesis para Bauman) de la cual cerecerían en el mundo análogo, las habilidades personales para enfrentarse a la cotidianidad. En psiquiatría, estos sujetos son denominados hikikomoris, palabra japonesa para definir la reclusión o el aislamiento, son quizá su antecedente el otaku ${ }^{6}$, individuos que escogen convertir ese mundo digital una realidad mejor que la que da el mundo analógico; identifican que la fuente de sus problemas es exactamente ese mundo analógico. De esta manera, son individuos con grandes habilidades en nuevas tecnologías de la información y con rápidos procesos cognitivos que les permiten absorber cantidades significativas de información.

5. Cúmulo de datos e información generada por uso y aplicación de las tecnologías digitales.

6. Tribu urbana que es fanática de la cultura de medios japoneses. 
Los aislamientos voluntarios, llamados screenagers, y otros sujetos similares, como los hikikimoris, son términos que definen a individuos que pasan largas horas frente a una pantalla de computadora o en el teléfono celular por situaciones de trabajo, laboratorios o instituciones académicas.

Estos individuos están sujetos al cambio para recobrar la realidad, podrían mezclar los mundos y perder la orientación de lo físico y digital; ya que al ser consumidores de información masiva, al minar ciertos datos se podrían entretejer factores sobre los valores y creencias de lo digital a lo físico.

Para tener una amplia visión caracterizada de los sujetos, en la tabla 1 se muestra y compara aspectos sociales de cada uno.

TABLA 1

Tipos sociales en sociedades digitales

\begin{tabular}{|c|c|c|c|c|}
\hline \multirow[b]{2}{*}{ Grupos } & \multicolumn{2}{|c|}{ Relaciones sociales análogas } & \multicolumn{2}{|c|}{ Relaciones sociales digitales } \\
\hline & $\begin{array}{l}\text { Ermitaños } \\
\text { digitales }\end{array}$ & Usuarios moderados & Screeners & Hikikimoris \\
\hline $\begin{array}{l}\text { Relaciones sociales en } \\
\text { sociedades digitales }\end{array}$ & $\begin{array}{l}\text { Mínimo o nulo } \\
\text { contacto digital }\end{array}$ & $\begin{array}{l}\text { Utilizan los medios } \\
\text { como herramientas } \\
\text { tipo feed news, y } \\
\text { contenido } \\
\text { multimedial }\end{array}$ & $\begin{array}{l}\text { Generalmente } \\
\text { las herramientas } \\
\text { digitales le } \\
\text { mantienen en } \\
\text { sociedades digitales } \\
\text { por razones } \\
\text { laborales }\end{array}$ & Inmersión digital \\
\hline $\begin{array}{l}\text { Relaciones sociales en } \\
\text { sociedades análogas }\end{array}$ & Inmersión social & $\begin{array}{l}\text { Relaciones sociales } \\
\text { entre iguales }\end{array}$ & $\begin{array}{l}\text { Relaciones } \\
\text { sociales } \\
\text { a nivel laboral }\end{array}$ & $\begin{array}{l}\text { Sin relaciones } \\
\text { sociales } \\
\text { significativas }\end{array}$ \\
\hline Tipo de interacción social & $\begin{array}{l}\text { Sin interacción } \\
\text { digital }\end{array}$ & $\begin{array}{l}\text { Debates sociales e } \\
\text { interacciones } \\
\text { casuales }\end{array}$ & $\begin{array}{l}\text { - Interacciones labor } \\
\text { financiero, académ } \\
\text { (freelance) } \\
\text { - Interacciones digita }\end{array}$ & $\begin{array}{l}\text { rales a nivel } \\
\text { hico o de servicios } \\
\text { ales intensas }\end{array}$ \\
\hline Relaciones económicas & $\begin{array}{l}\text { Pago mediante } \\
\text { dinero en efectivo }\end{array}$ & $\begin{array}{l}\text { Pago mediante dinero } \\
\text { medios digitales como }\end{array}$ & $\begin{array}{l}\text { en efectivo y por } \\
\text { transferencias }\end{array}$ & $\begin{array}{l}\text { Pago mediante } \\
\text { transferencias } \\
\text { bancarias o uso de } \\
\text { criptomonedas }\end{array}$ \\
\hline Relaciones culturales & $\begin{array}{l}\text { Asistencia a espect } \\
\text { actividades al aire }\end{array}$ & $\begin{array}{l}\text { áculos, exposiciones, sen } \\
\text { ibre }\end{array}$ & minarios y & $\begin{array}{l}\text { Asistencia a } \\
\text { actividades digitales } \\
\text { como torneos, } \\
\text { webinars y sesiones } \\
\text { remotas }\end{array}$ \\
\hline
\end{tabular}

Fuente: Elaboración propia.

Este espacio digital se convirtió en un mundo paralelo donde estos sujetos trabajan, estudian, se divierten, tienen familia y construyen otros espacios para desarrollar nuevas estructuras sociales. Esta interacción muestra la necesidad de expandir esa prótesis definida por Bauman. 
Manuel Castells mencionaba, de manera visionaria, que esta sociedad debe apuntar a ser un espacio de bien público, construido por todos; no como una mercancía, sino en el proceso social colectivo y un conocimiento social atemporal y sin más espacio que dado por la red, una construcción social sin dueño y sin objetivo definido. Muy probablemente los cambios sociales más sobresalientes de este nuevo siglo van a ser la batalla de las ideologías «del deber ser» de una sociedad, ya que como estos espacios son contruidos por diferentes sujetos sociales que tienen valores e ideologías muy distintas entre sí, se construye un ser social atemporal y contracultural que no respeta el statu quo ni el establishment ideológico, sino que construye uno particular, híbrido y subjetivo.

Esta batalla de los nuevos sujetos sociales en la sociedad digital contra los que quieren transmitir mensajes que influyan en las decisiones en la sociedad análoga, es la batalla que actualmente se da entre el plano objetivo de la realidad donde se crea el poder $\mathrm{y}$ en el espacio en donde se deciden las relaciones de poder, contra un plano subjetivo de la realidad que construye un nuevo poder y un nuevo espacio donde se decide el cómo de las relaciones de poder.

Ejemplos de ello hay muchos, como el poder real de la Casa Blanca con el presidente Trump, que lucha con esa realidad subjetiva que destila en los espacios virtuales, o como naciones enteras que deben lidiar con la construcción de sujetos sociales en el mundo digital que pueden terminar con países enteros, como el reciente ejemplo de las llamadas primaveras en territorios del norte de África y Medio Oriente. Hoy se denuncia por medio de los espacios virtuales y se opina construyendo socialmente desde lo virtual a elementos de la realidad objetiva.

\section{A manera de cierre}

Esta es la ciudadanía digital, la que rehúye de las instituciones políticas desgastadas con un modelo democrático erosionado por la corrupción y el abuso de poder; no solo desde los países desarrollados, sino en muchos países subdesarrollados, la cual se afirma en espacios de construcción, de movilización social, todo en sus propioss términos, y que entra y sale del sistema político formal; que además cuenta con una resistencia que realiza una reingeniería del sujeto social; con la creencia de cambiar la realidad objetiva.

Esta es la esencia de los nuevos actores sociales que pueden llevar a cabo el cambio social en la reconstruccion del las redes de comunicación y los espacios sociales, así como la reprogramación de los códigos culturales, los valores e intereses sociales y políticos que nacen de la desconfianza de los medios de comunicación tradicionales y el acceso irrestricto de nuevas formas de ver la realidad, con lo cual se genera la posibilidad de un nuevo contrato social que imponga nuevas relaciones de poder.

Se debe aclarar que no se trata solo de ciberactivistas, sino que los constructos sociales desde los cuales parten en la realidad objetiva, se subjetivizan con actores sociales de otras culturas, no solamente con actores de países capitalistas, sino también con países socialistas; no solo con actores de sociedades occidentales, sino con asiáticos o africanos o árabes. Este intercambio genera un desgaste importante en los mecanismos de hegemonía cultural que suelen existir en las clases dominantes; surge de la dialéctica entre 
la dominación, las resistencias y las alternativas que se contruyen espontáneamente y mediante la asociación libre de sujetos y movimientos sociales organizados.

El ermitaño se mantiene al margen de estos cambios. Es importante porque son los últimos vestigios de una era que se meteoriza; el ser humano tiene unas cualidades intrínsecas que, como se mencionaba al inicio, ha modificado su ambiente para luchar por su sobrevivencia, pero en especial para recrearse a sí mismo de manera indefinida; es la forma que tiene el humano para perpetuarse, solo mediante una crisis la sociedad puede ver el futuro.

El ermitaño evita el uso de las herramientas que generan las nuevas tecnologías, las que generarían un nuevo modelo social, cuya base está construida sobre el componente tecnológico. Pero es inevitable que los motores de desarrollo de esos capitales sociales se den, son diversos y generan riqueza, pero todos son subjetivos, intangibles, su peso social se ve en los diferentes servicios de las empresas y en los productos simbólicos que, con avidez, los sujetos sociales consumen por ejemplo: aplicaciones para tabletas y celulares, elementos de juegos como escudos, espadas, ropa para sus avatares o complementos.

A esta altura no se podría definir si estos sujetos son nativos digitales o migrantes; o, por otra parte, determinar a cuál generación pertenecen (baby boomers, Generación X, millennials y centennials). Así, la temporalidad no es importante para ser o no ermitaño dentro de la aldea global digital.

\title{
ABSTRACT
}

The social basis of the global village. Approaches to understand the nature of the virtual world

\begin{abstract}
Based on critical theory, this essay proposes a vision of the new citizenship and the society that has been configured with the appearance of digital media while at the same time establishing certain behaviors in the light of some of the theoretical issues and from the Western viewpoint of this phenomenon. The aspects are divided in four large groups regarding social media use in the global village. In addition, it suggests a way to define the social structure of digital societies from critical epistemology.
\end{abstract}

Key words: Information society, digital hermits, screenagers, hikikimoris.

RÉSUMÉ

La base sociale du village planétaire. Approches pour comprendre la nature du monde virtuel

Cet essai propose, à partir de la théorie critique, une perspective de la nouvelle citoyenneté et de la société qui a été créée avec les médias numériques. En outre, il a pour but d'établir certains comportements à la lumière des fondements théoriques et de la vision occidentale de ce phénomène. Ces aspects sont perceptibles dans les quatre grands groupes dans les réseaux sociaux qui conforment le village planétaire. D'ailleurs, l'essai offre une manière de définir la structure sociale des sociétés numériques dès l'épistémologie critique.

Mots-clés: Société de l'information, ermite numérique, screenagers (jeunes adeptes de l'écran), hikikomoris. 


\section{Referencias bibliográficas}

Bauman, Zygmunt. Amor líquido: acerca de la fragilidad de los vínculos humanos. México: Fondo de Cultura Económica, 2012.

Bauman, Zygmunt. «The sweet scent of decomposition», en Forget Baudrillard por ed. Chris Rojek. Londres: Routledge, 1993.

Bourdieu, Pierre. Capital cultural, escuela y espacio social. Buenos Aires: Siglo XXI, 2005.

Bourdieu, Pierre. Campos del poder y campo intelectual. Buenos Aires: Montressor Jungla Simbólica, 2002.

Castells, Manuel. La era de la información: la sociedad red. Madrid: Alianza Editorial, 2005.

Castells, Manuel. The Internet Galaxy: reflections on the Internet, Business, and Society. Oxford: University Press, 2001.

Díez Gutiérrez, Enrique. «Reseña de "Comunicación y poder" de Castells». Revista Interuniversitaria de Formación del Profesorado, 25, 2 (2011). Acceso: 03 de diciembre, 2019. http://www.redalyc.org/ pdf/274/27422047013.pdf

Gracía, Paula. «Los smartphones: La droga invisible del siglo XXI» (2018). Acceso: 03 de diciembre, 2019. https://ddd.uab.cat/record/196460

Joignant, Alfredo. «Hábitus, campo y capital. Elementos para una teoría de capital político». Revista Mexicana de Sociología, 4 (2012). Acceso: 03 de diciembre, 2019. http://www.scielo.org.mx/scielo.php?script=sci_ar ttext\&pid=S0188-25032012000400003

Morente, Manuel. Lecciones preliminares de filosofía. México: Editorial Porrúa, 2001.

Trujano, Patricia. «Hikikomoris y screenagers: nuevas formas de reclusión, inhibición y aislamiento». Revista electrónica Interpsiquis, 10 (2009). Acceso: 03 de diciembre, 2019. https://psiquiatria.com/bibliopsiquis/ hikikomoris-y-screenagers-nuevas-formas-de-reclusion-inhibicion-y-aislamiento/ 